\title{
Stereotactic radiosurgery for patients with multiple brain metastases: a case-matched study comparing treatment results for patients with $2-9$ versus 10 or more tumors
}

\author{
Clinical article
}

\author{
Masaaki Yamamoto, M.D., ${ }^{1,2}$ Takuya Kawabe, M.D., ${ }^{1,3}$ Y ASunori Sato, PH.D., ${ }^{4}$ \\ Yoshinori Higuchi, M.D., ${ }^{5}$ Tadashi Nariai, M.D., Ph.D., ${ }^{6}$ Shinya Watanabe, M.D., ${ }^{1,7}$ \\ AND Hidetoshi Kasuya, M.D. ${ }^{2}$
}

\begin{abstract}
${ }^{I}$ Katsuta Hospital Mito GammaHouse, Hitachi-naka; ${ }^{2}$ Department of Neurosurgery, Tokyo Women's Medical University Medical Center East, Tokyo; ${ }^{3}$ Department of Neurosurgery, Kyoto Prefectural University of Medicine Graduate School of Medical Sciences, Kyoto $;{ }^{4}$ Clinical Research Center, Chiba University Graduate School of Medicine, Chiba; ${ }^{5}$ Department of Neurosurgery, Chiba University Graduate School of Medicine, Chiba; ${ }^{6}$ Department of Neurosurgery, Graduate School, Tokyo Medical and Dental University School of Medicine, Tokyo; and ${ }^{7}$ Department of Neurosurgery, Faculty of Medicine, University of Tsukuba, Tsukuba, Japan
\end{abstract}

\begin{abstract}
Object. Although stereotactic radiosurgery (SRS) alone is not a standard treatment for patients with 4-5 tumors or more, a recent trend has been for patients with 5 or more, or even 10 or more, tumors to undergo SRS alone. The aim of this study was to reappraise whether the treatment results for SRS alone for patients with 10 or more tumors differ from those for patients with 2-9 tumors.

Methods. This was an institutional review board-approved, retrospective cohort study that gathered data from the Katsuta Hospital Mito GammaHouse prospectively accumulated database. Data were collected for 2553 patients who consecutively had undergone Gamma Knife SRS alone, without whole-brain radiotherapy (WBRT), for newly diagnosed (mostly) or recurrent (uncommonly) brain metastases during 1998-2011. Of these 2553 patients, 739 (28.9\%) with a single tumor were excluded, leaving 1814 with multiple metastases in the study. These 1814 patients were divided into 2 groups: those with 2-9 tumors (Group A, 1254 patients) and those with 10 or more tumors (Group B, 560 patients). Because of considerable bias in pre-SRS clinical factors between groups A and B, a case-matched study, which used the propensity score matching method, was conducted for clinical factors (i.e., age, sex, primary tumor state, extracerebral metastases, Karnofsky Performance Status, neurological symptoms, prior procedures [surgery and WBRT], volume of the largest tumor, and peripheral doses). Ultimately, 720 patients (360 in each group) were selected. The standard Kaplan-Meier method was used to determine post-SRS survival times and post-SRS neurological death-free survival times. Competing risk analysis was applied to estimate cumulative incidence for local recurrence, repeat SRS for new lesions, neurological deterioration, and SRS-induced complications.

Results. Post-SRS median survival times did not differ significantly between the 2 groups (6.8 months for Group A vs 6.0 months for Group B; hazard ratio [HR] 1.133, 95\% CI 0.974-1.319, p = 0.10). Furthermore, rates of neurological death were very similar: $10.0 \%$ for group A and $9.4 \%$ for group B $(p=0.89)$; neurological death-free survival times did not differ significantly between the 2 groups (HR $1.073,95 \%$ CI $0.649-1.771, \mathrm{p}=0.78$ ). The cumulative incidence of local recurrence (HR $0.425,95 \%$ CI $0.0 .181-0.990, p=0.04$ ) and repeat SRS for new lesions (HR 0.732, 95\% CI 0.554-0.870, p = 0.03) were significantly lower for Group B than for Group A patients. No significant differences between the groups were found for cumulative incidence for neurological deterioration (HR 0.994, 95\% CI 0.607-1.469, p = 0.80) or SRS-related complications (HR $0.541,95 \%$ CI $0.138-2.112, \mathrm{p}=0.38)$.

Conclusions. Post-SRS treatment results (i.e., median survival time; neurological death-free survival times; and cumulative incidence for local recurrence, repeat SRS for new lesions, neurological deterioration, and SRS-related complications) were not inferior (neither less effective nor less safe) for patients in Group B than for those in Group A. We conclude that carefully selected patients with 10 or more tumors are not unfavorable candidates for SRS alone. A randomized controlled trial should be conducted to test this hypothesis.
\end{abstract}

(http://thejns.org/doi/abs/10.3171/2014.8.GKS141421)

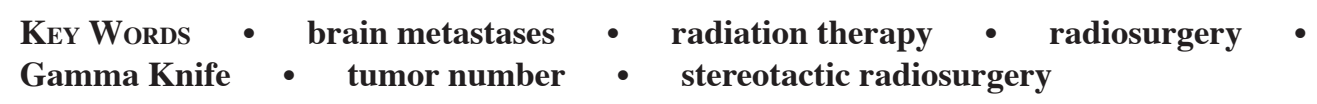

\footnotetext{
Abbreviations used in this paper: $\mathrm{HR}=$ hazard ratio; $\mathrm{KPS}=$ Karnofsky Performance Status; RPA = recursive partitioning analysis; $\mathrm{SRS}=$ stereotactic radiosurgery; $\mathrm{WBRT}=$ whole-brain radiation therapy.
}

$\mathrm{S}$ INCE Sturm et al. reported successful treatment of brain metastases with use of stereotactic radiosurgery (SRS), ${ }^{25}$ evidence of the effectiveness of this treatment strategy has been accumulating, for both SRS 


\section{Radiosurgery for $2-9$ versus 10 or more metastatic brain tumors}

alone and SRS in combination with whole-brain radiotherapy (WBRT). ${ }^{1,3,10,13,15,23,24}$ Among patients with brain metastases, numerous factors affect outcomes, such that a one-size-fits-all treatment paradigm is no longer appropriate. Nevertheless, a solid patient selection criterion is still necessary before SRS treatment of brain metastases. SRS alone for patients with 4 or more, or even 5 or more, metastatic tumors is not a standard treatment, and in most industrialized nations, WBRT is still strongly recommended..$^{17}$ However, by the early 21 st century, a trend for patients with 5 or more tumors to be potential candidates for SRS alone had already become apparent. $3,8,13,15,16,20,23,24,26-30,32$ Very recently, in a prospective observational study of 1194 brain metastasis patients, Yamamoto et al. showed that SRS without WBRT as the initial treatment was not inferior (in terms of overall survival as well as most secondary end points) for patients with 5-10 metastatic brain tumors compared with those with 2-4 metastatic tumors. ${ }^{35}$ Considering the present lack of evidence supporting the superiority of WBRT over SRS alone for patients with 5-10 tumors, their results are considered to constitute the highest level of evidence to date, allowing SRS alone to be advocated for such patients. Existing treatment guidelines for managing brain metastasis patients need to be revised in the very near future.

The next step is to reappraise whether SRS alone is inferior for patients with 10 or more metastatic tumors compared to patients with fewer metastatic tumors. Very recently, 2 studies evaluating outcomes in patients with 10 or more metastatic brain tumors treated with SRS were published in the Journal of Neurosurgery. ${ }^{8,20}$ Grandhi et al. studied 61 patients with 10 or more metastatic brain tumors (mean 13 tumors) treated with SRS and found median survival time after SRS to be 4 months; they concluded that SRS safely and effectively treats intracranial disease with a high rate of local control in patients with 10 or more metastatic brain tumors. ${ }^{8}$ These authors stated that SRS might be one of the most effective treatment options available for patients with fewer tumors, a non-melanomatous primary lesion, controlled systemic disease, and assignment to a low recursive partitioning analysis (RPA) class. Grandhi et al. concluded that SRS can thus be regarded as a first-line treatment. Rava et al. studied 53 patients with 10 or more metastatic brain tumors treated with SRS (mean number of tumors = 11) and found that post-SRS median survival time exceeded 6 months; they concluded that aggressive local treatment remains an option, although rapid CNS failure is to be expected. ${ }^{20}$ Patients with breast cancer represent a group of patients likely to benefit greatly from SRS alone; survival time and time to CNS recurrence are extended. Although these 2 articles discuss several important issues and present significant potential treatment advances, a common weakness is that sample sizes were relatively small (61 and 53 patients). 8,20

Therefore, we conducted this retrospective cohort study, based on our SRS-treated brain metastasis patients, including 560 patients with 10 or more tumors, to reappraise whether treatment results were truly inferior for patients with 10 or more versus 2-9 tumors and to identify factors determining inferiority and/or noninferior- ity. We excluded patients with only 1 tumor because it is widely recognized that survival periods are much longer for patients with a single brain metastastic tumor than for those with multiple metastatic brain tumors..$^{13,23,24,30}$

\section{Methods}

\section{Patient Population}

This retrospective cohort study used the prospectively accumulated Katsuta Hospital Mito GammaHouse database of 2553 patients who consecutively underwent SRS alone, without WBRT, for newly diagnosed (mostly) or recurrent (uncommonly) brain metastases during the 13-year-period July 1998 through June 2011. The study was approved by the institutional review board of Tokyo Women's Medical University. Because all patients had been referred to the Katsuta Hospital Mito GammaHouse for SRS, their primary physicians had made most of the patient selections. Patient selection criteria may thus have differed among referring physicians. Therefore, one author (M.Y.) decided whether to accept a patient. We did not perform SRS on patients with low Karnofsky Performance Status (KPS) scores ${ }^{14}$ resulting from systemic diseases, a noncooperative state resulting from poor neurocognitive function, meningeal dissemination, or an anticipated survival period of 3 months or less. Therefore, only $173(6.8 \%)$ patients were categorized into RPA Class III, ${ }^{6}$ while $1189(46.6 \%)$ were categorized into modifiedRPA Class IIc+III. ${ }^{33,34}$ Also, the primary physicians responsible for each patient decided the indications for both surgery and radiotherapy. Therefore, before undergoing SRS, $468(18.3 \%)$ of the 2553 patients had undergone surgical removal of brain metastases and 125 (4.9\%) had undergone WBRT.

Before SRS, the first author (M.Y.) explained the treatment strategy in detail to each patient and at least 1 adult relative of the patient. Written informed consent was obtained from all patients. Because our previous report ${ }^{32,33}$ describes our radiosurgical techniques and dose selection for multiple brain metastases in detail, they are not repeated herein. In brief, before June 2003, standard SRS procedures were performed by using a Leksell Gamma Unit Model B and thereafter a Leksell Gamma Unit Model $\mathrm{C}$ (Elekta $\mathrm{AB}$ ). For target coordinate determination and dose planning, until August 2002 stereotactic single-dose gadolinium-enhanced T1-weighted axial MR images with a slice thickness of $2 \mathrm{~mm}$, multiple slices of which covered the entire brain, were obtained by using a Magnetom Impact Expert 1.0-T unit (Siemens); thereafter, a Magnetom Symphony 1.5-T unit (Siemens) was used.

After SRS, patients were usually managed by their referring physicians; clinical and neuroimaging examinations at approximately 2 - to 3 -month intervals were recommended. However, for 761 (29.8\%) of the 2553 patients, neuroimaging follow-up could not be performed because of early post-SRS death or remarkable deterioration of general condition. Among the 2553 patients, follow-up information was obtained during periodic visits to our outpatient clinic (approximately 60\%), from clinical and/ or neuroimaging data that were mailed to us (about 25\%), 
or by phone call from the first author (M.Y.) to patients or their relatives $(15 \%)$. For deceased patients, information about the day of death, cause of death, and detailed information on patient condition changes was obtained by telephone calls to relatives.

\section{Study Design and Case Matching}

Karlsson et al. ${ }^{13}$ recently conducted a study of 1921 brain metastasis patients who underwent SRS. They reported that although patients with a single metastatic brain lesion survived longer than those with multiple brain metastases, median survival times did not significantly differ among patients with 2, 3-4, 5-8, or more than 8 metastatic tumors. Also, using our database and including the present cohort (of the 2553 patients, 2232 had 15 tumors or fewer), we used the Kaplan-Meier meth$\mathrm{od}^{12}$ to compare 14 pairs of groups based on number of tumors (1 tumor vs 2 tumors, 2 vs 3,3 vs 4 , and so on through 14 vs 15). As shown in Table 1, among the 14 pairs, median survival times differed significantly only for patients with 1 versus 2 tumors ( $\mathrm{p}<0.001$ ); no significant differences were detected for the other 13 pairs of groups based on number of tumors (Bonferroni threshold $0.05 / 14=0.0036)$. Thus, we excluded $739(28.9 \%)$ patients with a single metastatic brain lesion and studied the remaining 1814 patients with multiple metastatic brain tumors. These 1814 patients were divided into 2 groups; those with 2-9 tumors (Group A, 1254 patients) and those with 10 or more tumors (Group B, 560 patients).

Because there was bias and a large discrepancy in the number of patients in groups A and B, a case-matched study was conducted by one of the authors (Y.S.), who did not participate in other aspects of this study and was blinded as to the final outcomes. Patient selection was performed by using the propensity score matching method with a Greedy 5-To-1 Digit-Matching algorithm ${ }^{18}$ for clinical factors (i.e., age, sex, primary tumor status, extracerebral metastases, KPS score, neurological symptoms, prior procedures [surgery and WBRT], volume of the largest tumor, and peripheral doses). ${ }^{4,21}$ After all the propensity score matches had been performed, we compared baseline covariates between the 2 groups. Ultimately, 720 patients were selected (360 with 2-9 tumors [Group A] and 360 with 10 or more tumors [Group B]) (Table 2). ${ }^{33,34}$ The $\mathrm{p}$ values after matching exceeded 0.05 for all clinical factors.

\section{Clinical Outcomes}

The primary end point was overall survival, and the secondary end points were neurological death, neurological deterioration, local recurrence of the treated tumor, repeat SRS for new lesions, and SRS-induced major complications. For each end point, failures were regarded as events, and any others were regarded as censored. Because the criteria for each end point have been described, ${ }^{32,33}$ they are not repeated herein.

\section{Statistical Analyses}

All data were analyzed according to the intentionto-treat principle. For the baseline variables, summary statistics were constructed by using frequencies and proportions for categorical data and using means and standard deviations for continuous variables. We compared patient characteristics by using the Fisher exact test for categorical outcomes and t-tests for continuous variables, as appropriate. The standard Kaplan-Meier method was used for overall and neurological death-free survival calculations. ${ }^{12}$ Also, to determine pre-SRS clinical factors favoring longer survival time, we performed univariable analysis by using the Cox proportional hazard model.

For time-to-event outcomes, we estimated the cumulative incidence for local recurrence, repeat SRS, neuro-

TABLE 1: Median survival time differences between patient groups based on number of tumors*

\begin{tabular}{ccccc}
\hline No. of Tumors & No. of Patients & Median Survival Time $(95 \% \mathrm{Cl})$, Months & HR $(95 \% \mathrm{Cl})$ & Log-Rank p Value $\dagger$ \\
\hline 1 & 739 & $10.6(9.4-11.8)$ & & \\
2 & 390 & $7.4(6.7-8.4)$ & $0.730(0.641-0.831)$ & $<0.001$ (vs 1) \\
3 & 235 & $8.0(6.4-9.2)$ & $1.046(0.883-1.236)$ & 0.60 (vs 2) \\
4 & 189 & $7.1(5.9-8.5)$ & $0.994(0.815-1.216)$ & 0.96 (vs 3) \\
5 & 118 & $6.2(5.1-7.6)$ & $0.953(0.751-1.213)$ & 0.69 (vs 4) \\
6 & 115 & $7.0(4.8-9.1)$ & $1.030(0.789-1.345)$ & 0.83 (vs 5) \\
7 & 69 & $8.1(5.0-10.6)$ & $1.042(0.759-1.420)$ & 0.79 (vs 6) \\
8 & 80 & $5.5(3.2-6.5)$ & $0.656(0.468-0.916)$ & 0.01 (vs 7) \\
9 & 57 & $5.1(3.7-7.0)$ & $0.888(0.625-1.253)$ & 0.50 (vs 8) \\
10 & 69 & $7.0(4.3-9.3)$ & $1.186(0.824-1.700)$ & 0.35 (vs 9) \\
11 & 46 & $6.3(3.7-9.1)$ & $0.980(0.665-1.432)$ & 0.92 (vs 10) \\
12 & 32 & $7.1(3.5-10.7)$ & $1.063(0.676-1.694)$ & 0.79 (vs 11) \\
13 & 35 & $6.1(3.8-8.9)$ & $0.971(0.591-1.570)$ & 0.96 (vs 12) \\
14 & 38 & $5.7(2.6-10.0)$ & $0.936(0.580-1.504)$ & 0.78 (vs 13) \\
15 & 20 & $8.5(3.8-11.0)$ & $1.038(0.578-1.810)$ & 0.90 (vs 14) \\
\hline
\end{tabular}

* Data exclude 4 patients who were lost to follow-up.

$\dagger$ Parentheses indicate number of tumors in comparison group. 
Radiosurgery for 2-9 versus 10 or more metastatic brain tumors

TABLE 2: Summary of clinical characteristics of 720 patients with brain metastases*

\begin{tabular}{|c|c|c|c|c|}
\hline \multirow[b]{2}{*}{ Characteristic } & \multirow[b]{2}{*}{ Total } & \multicolumn{2}{|c|}{ No. of Tumors } & \multirow[b]{2}{*}{$p$ Value } \\
\hline & & 2-9 (Group A) & $\geq 10$ (Group B) & \\
\hline no. of patients & 720 & 360 & 360 & \\
\hline no. lost to follow-up & 3 & $1(0.3 \%)$ & $2(0.6 \%)$ & 1.00 \\
\hline \multicolumn{5}{|l|}{ no. of tumors } \\
\hline mean & 11 & 4 & 17 & \\
\hline median & 9,10 & 4 & 14 & \\
\hline range & $2-69$ & $2-9$ & $10-69$ & \\
\hline \multicolumn{5}{|l|}{ age (yrs) } \\
\hline mean & 63 & 63 & 63 & 0.98 \\
\hline range & $25-96$ & $26-96$ & $25-91$ & \\
\hline sex: female & $319(44.3 \%)$ & $154(42.8 \%)$ & $165(45.8 \%)$ & 0.45 \\
\hline \multicolumn{5}{|l|}{ primary cancer sites } \\
\hline lung & $498(69.2 \%)$ & $251(69.7 \%)$ & $247(68.6 \%)$ & $0.81 \ddagger$ \\
\hline breast & 101 (14.0\%) & 49 (13.6\%) & $52(14.4 \%)$ & \\
\hline alimentary tract & $61(8.5 \%)$ & $36(10.0 \%)$ & $25(6.9 \%)$ & \\
\hline kidney & $19(2.6 \%)$ & $7(1.9 \%)$ & $12(3.3 \%)$ & \\
\hline others & $41(5.7 \%)$ & $17(4.7 \%)$ & $24(6.7 \%)$ & \\
\hline primary cancer status: controlled & $195(27.1 \%)$ & $104(28.9 \%)$ & $91(25.3 \%)$ & 0.31 \\
\hline extracerebral METs: no. & $342(47.5 \%)$ & $169(46.9 \%)$ & $173(48.1 \%)$ & 0.82 \\
\hline KPS score $\geq 80 \%$ & $542(75.3 \%)$ & $269(74.7 \%)$ & $273(75.8 \%)$ & 0.80 \\
\hline \multicolumn{5}{|l|}{ modified-RPA class } \\
\hline I+lla & 76 (10.6\%) & 42 (11.7\%) & $34(9.4 \%)$ & \\
\hline $\mathrm{llb}$ & $220(30.6 \%)$ & $106(29.4 \%)$ & $114(31.7 \%)$ & $0.35 \S$ \\
\hline$\|c+I\|$ & $424(58.9 \%)$ & $212(58.9 \%)$ & $212(58.9 \%)$ & $0.68 \pi$ \\
\hline neurological Sx: no & $353(49.0 \%)$ & $176(48.9 \%)$ & $177(49.2 \%)$ & 1.00 \\
\hline prior surgery: yes & $103(14.3 \%)$ & $54(15.0 \%)$ & $49(13.6 \%)$ & 0.67 \\
\hline prior WBRT: yes & $36(5.0 \%)$ & $19(5.3 \%)$ & $17(4.7 \%)$ & 0.86 \\
\hline \multicolumn{5}{|l|}{ tumor volume $\left(\mathrm{cm}^{3}\right)$} \\
\hline \multicolumn{5}{|l|}{ cumulative } \\
\hline mean & 10.88 & 11.45 & 10.31 & 0.26 \\
\hline range & $0.08-115.3$ & $0.08-115.3$ & $0.15-81.4$ & \\
\hline \multicolumn{5}{|l|}{ largest tumor } \\
\hline mean & 6.14 & 6.51 & 5.77 & 0.24 \\
\hline range & $0.03-94.2$ & $0.04-94.2$ & $0.03-65.0$ & \\
\hline \multicolumn{5}{|l|}{ peripheral dose (Gy) } \\
\hline mean & 20.62 & 20.51 & 20.72 & 0.35 \\
\hline range & $10.00-25.00$ & $10.00-25.00$ & $12.00-25.00$ & \\
\hline
\end{tabular}

* KPS = Karnofsky Performance Status; METs = metastases; $\mathrm{Sx}=$ symptoms.

$\dagger$ The Student t-test was used for continuous variables and the Fisher exact test for pairs of categorical variables.

‡ Lung vs not lung.

$\S$ Modified RPA Class I+lla vs Ilb.

II Modified RPA Class Ilb vs IIc+III.

logical deterioration, and major complications by using a competing risk analysis because death is a competing risk for loss to follow-up (i.e., patients who die can no longer

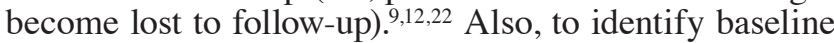
and clinical variables associated with the 4 above-mentioned outcomes, we performed competing risk analyses with the Fine-Gray generalization of the proportional hazards model accounting for death as a competing risk.$^{5,7}$ For modeling cumulative incidence, the Fine-Gray generalization uses the subdistribution hazard, thereby quantifying the overall benefit or harm of an exposure. ${ }^{2}$

All comparisons were planned, and the tests were 2 -sided. A p value of less than 0.05 was considered to be statistically significant. All statistical analyses were performed by one of the authors (Y.S.) who used SAS software, version 9.3 (SAS Institute), and the R statistical 
program, version 3.0.0. Before statistical analyses, inaccurate records in the database were corrected (by Y.H.). These 2 authors were not involved in either SRS treatment or patient follow-up evaluations.

\section{Results}

Four patients were lost to follow-up and excluded from analysis, leaving a cohort of 2549 patients. Among these 2549 patients, the overall post-SRS median survival time was 7.4 (95\% CI 7.1-7.9) months. In the subset of 720 patients (excluding 3 patients lost to follow-up [1 in Group A and 2 in Group B]), the median post-SRS follow-up time among censored observations (39 patients) was 16.7 (range 2.1-110.2) months, and 678 (94.2\%) patients died. The post-SRS median survival time was 6.4 (95\% CI 5.7-7.0) months. Actuarial survival rates after SRS were $51.9 \%, 27.7 \%, 10.5 \%, 4.3 \%$, and $1.6 \%$ at 6,12 , 24,36 , and 60 months, respectively. For 40 patients who died, the cause of death could not be determined; but for the remaining 638, the cause of death was confirmed to be non-brain diseases for $576(90.3 \%)$ and brain diseases for $62(9.7 \%)$. Among the subset of 720 patients, salvage surgery was required for 4 patients and WBRT for 29 patients. There were no significant differences between the 2 groups in the incidences of salvage surgery $(0.6 \%$ vs $0.6 \%, \mathrm{p}=1.00)$ and WBRT $(3.1 \%$ vs $5.0 \%, \mathrm{p}=0.19)$ (Table 3).

\section{Comparisons Between Groups A and B}

The post-SRS median survival time was slightly longer for patients in Group A (6.8 months) than for those in Group B (6.0 months). However, the median survival time difference, 0.8 months, did not reach statistical significance, as shown in the graph on the left side of Fig. 1 (hazard ratio [HR] 1.133, 95\% CI 0.975-1.320, $\mathrm{p}=0.10$ ). Incidence rates for death caused by brain disease progression were very similar: $10.0 \%$ for Group A and $9.4 \%$ for Group B ( $p=0.89)$ (Table 3). Furthermore, neurological death-free survival intervals did not differ significantly between the 2 groups (HR 1.059, 95\% CI 0.680-1.649, $\mathrm{p}$ $=0.80$ ) (Fig. 1 right).
Post-SRS follow-up MR images were available for 485 (67.4\%) patients: 249 (69.2\%) in Group A and 236 $(65.6 \%)$ in Group B $(\mathrm{p}=0.34)$. The median survival time for patients in whom follow-up MRI examinations were not available was 2.3 (95\% CI 1.8-2.6) months. Therefore, most patients in this group died or deteriorated remarkably because of extracerebral disease progression before post-SRS MRI examinations could be performed. Among these 485 patients, the incidence of local recurrence was significantly lower for those in Group B than in Group A $(3.0 \%$ vs $8.4 \%, p=0.01)$ (Table 3$)$. Also, the cumulative incidence of local recurrence differed significantly between the 2 groups (HR $0.425,95 \%$ CI $0.181-0.990, \mathrm{p}$ $=0.04)$ (Fig. $2 \mathrm{~A}$ ). Our criteria for detecting local recurrence by using MRI and/or methionine positron emission tomography are detailed elsewhere. ${ }^{31,32}$

As shown in Table 3, among the 720 patients, the incidence of repeat SRS for new lesions was significantly lower among patients in Group B than in Group A $(23.7 \%$ vs $32.3 \%, p=0.01)$. Also, there was a significant difference between the 2 groups in the cumulative incidence of repeat SRS for new lesions (HR 0.732, 95\% CI $0.554-0.970, p=0.03$ ) (Fig. 2B). There were no significant differences between the 2 groups in the incidence of neurological deterioration or SRS-related complications. Also, there were no significant differences between the 2 groups in the cumulative incidence of neurological deterioration (HR 0.994, 95\% CI 0.607-1.469, p = 0.80) (Fig. 2C) or SRS-related complications (HR 0.541, 95\% CI $0.138-2.112, \mathrm{p}=0.38$ ) (Fig. 2D).

\section{Factors Affecting Longer Survival for Patients With 10 or More Metastatic Brain Tumors}

As shown in Table 4, univariable analysis demonstrated that among various pre-SRS clinical factors, significant predictors of a longer survival period for the 360 patients with 10 or more metastatic brain tumors were female sex, younger age, controlled primary cancer, no extracerebral metastases, better KPS score, better modifiedRPA class, smaller tumor volume, and higher peripheral dose. Among these 8 factors, hazard ratios were higher for patients with a KPS score of $80 \%$ or higher versus

TABLE 3: Crude incidence of outcomes after SRS*

\begin{tabular}{|c|c|c|c|c|}
\hline Outcome & Total & 2-9 Tumors (Group A) & $\geq 10$ Tumors (Group B) & $p$ Value \\
\hline no. of patients & 720 & 360 & 360 & \\
\hline neurological death $\dagger$ & $62(9.7)$ & $32(10.0)$ & $30(9.4)$ & 0.89 \\
\hline salvage WBRT & $29(4.0)$ & $11(3.1)$ & $18(5.0)$ & 0.19 \\
\hline salvage surgery & $4(0.6)$ & $2(0.6)$ & $2(0.6)$ & 1.00 \\
\hline local recurrence $\ddagger$ & $28(5.8)$ & $21(8.4)$ & $7(3.0)$ & 0.01 \\
\hline repeat SRS & $201(27.9)$ & $116(32.3)$ & $85(23.6)$ & 0.01 \\
\hline neurological deterioration & $81(11.3)$ & $45(12.5)$ & $36(10.0)$ & 0.35 \\
\hline SRS-related complications & $12(1.7)$ & $9(2.5)$ & $3(0.8)$ & 0.14 \\
\hline
\end{tabular}



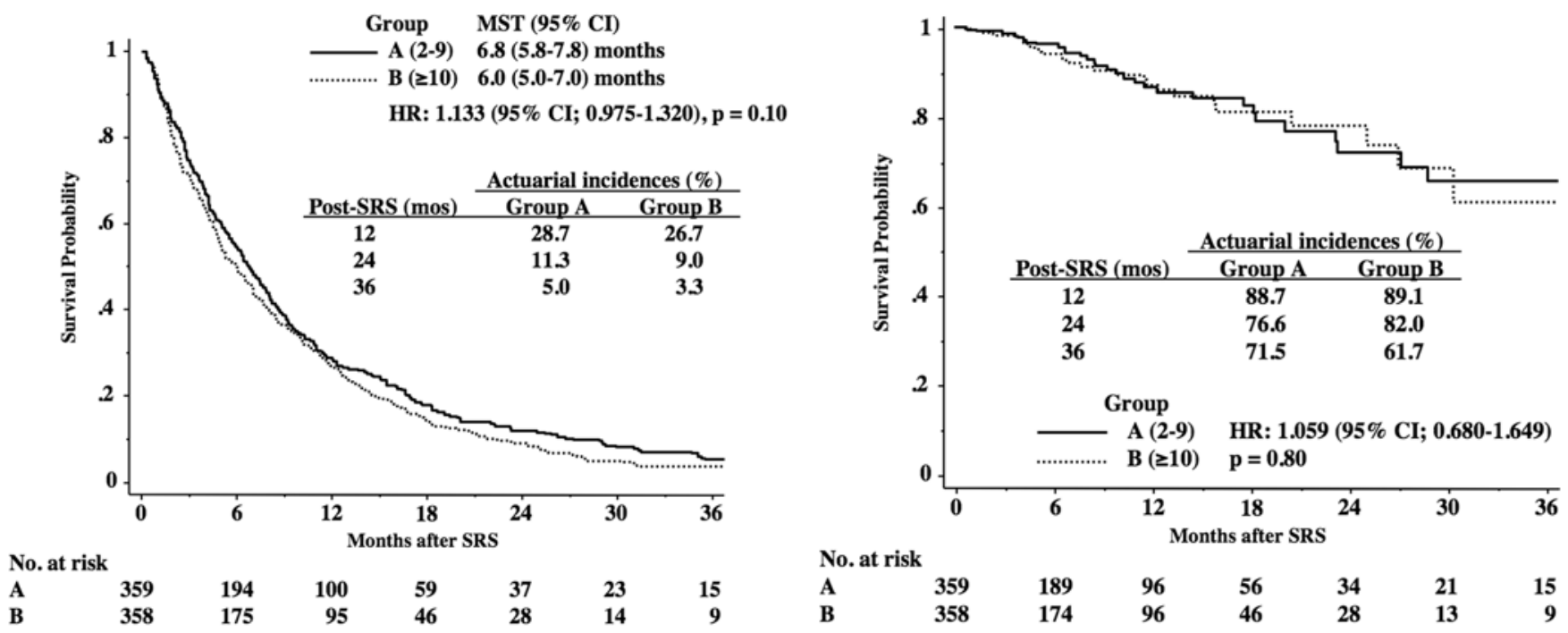

Fig. 1. Probabilities of overall survival (left) and neurological death-free survival (right) according to number of tumors (2-9 [Group A] and 10 or more [Group B]), estimated by using the Kaplan-Meier method.12 Three cases lost to follow-up were excluded. MST = median survival time.

$70 \%$ or lower, primary cancer controlled versus not controlled, no extracerebral metastases versus extracerebral metastases, and modified-RPA Class I+IIa versus IIb and Class IIb versus IIc+III. Also, as shown in Table 4, hazard ratios and 95\% confidence intervals for these 4 clinical factors were nearly the same for patients in groups A and B. All of the above-mentioned results differed minimally between the 2 groups.

\section{Discussion}

In the study reported here, the post-SRS median survival time difference between the 2 groups, 0.8 months, was not statistically significant. Furthermore, approximately $90 \%$ of patients with brain metastases died of causes other than brain disease progression, regardless of the number of metastatic tumors. The results for Group B patients were not inferior to those for Group A patients in terms of neurological death, local recurrence, repeat SRS required for new tumors, maintenance of good neurological state, and SRS-related complications. Because approximately $90 \%$ of patients died of extracerebral diseases, it is clearly crucial that brain metastasis treatments contribute to maintenance of a good neurological state. We thus consider it to be very important that the hereinreported results of maintenance of a good neurological state for patients with 10 or more tumors were clearly not inferior to results for patients with 2-9 tumors. Cumulative incidence rates of local recurrence and repeat SRS for patients in Group A were rather high compared with those for patients in Group B; these differences reached statistical significance, although the reasons for their significance are unclear. However, considering our large patient numbers and the fact that the upper $95 \%$ CIs $(0.990$ and 0.970$)$ were very close to $1.0, \mathrm{p}$ values $(0.04$ and 0.03$)$ were relatively high. Therefore, these differences are not considered to be particularly crucial.

Because we have extensively discussed the benefits of
SRS alone versus WBRT with or without SRS for patients with multiple brain metastases in 2 previous articles, ${ }^{32,35}$ we will not repeat them here. However, it is noteworthy that previously published studies show tumor numbers to not significantly affect post-SRS overall survival., ${ }^{3,13,30,32}$ The central criticism of SRS alone for treatment of multiple brain metastases is the assumption that frequent microscopic tumors will soon require salvage SRS or other treatments. Thus, WBRT has generally been advocated. However, as reported by Aoyama et al., the longest time that WBRT can be expected to prevent new tumors from arising is 6-8 months. ${ }^{1}$ We should remember that considerable numbers of patients with brain metastasis can survive more than 1 year, thereby outliving the effects of WBRT. Fortunately, we already live in an era when a metastatic brain lesion of $0.005 \mathrm{~cm}^{3}$ or even slightly smaller can be detected with thin-slice, gadolinium-enhanced MR images. ${ }^{11}$ Hanssens et al. recently reported that according to high-resolution MR imaging, SRS alone decreased the incidence of and lengthened the time to distant recurrences. ${ }^{10}$ Although data on periods between SRS and the appearance of new lesions were not available in the study reported here, the rate of repeat SRS among Group B patients was not inferior to that among Group A patients. Therefore, the availability of an alternative treatment for multiple brain metastases enables reservation of WBRT for subsequent treatment attempts (i.e., for meningeal dissemination or miliary metastases treatable only with WBRT).

\section{How Many Brain Metastases Make a Patient Ineligible for SRS?}

Current evidence-based guidelines have supported the use of SRS for patients with 1-4 metastatic brain tumors. ${ }^{17}$ However, such guidelines frequently lag behind contemporary clinical practice because at least several years are required for completion of rigorous prospective clinical trials. Particularly with use of SRS with a Gamma Knife, when targeting multiple tumors, each addition- 
A
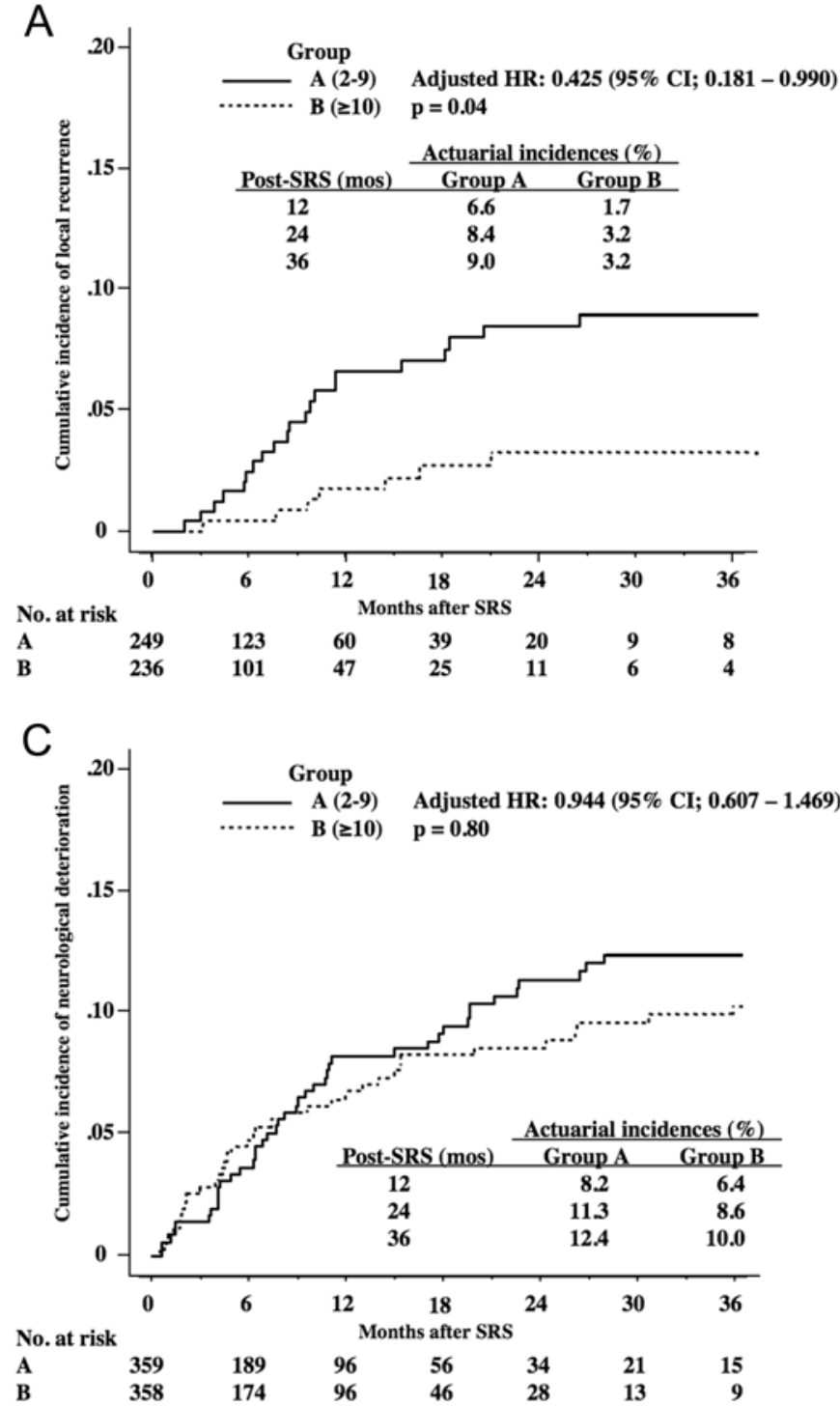
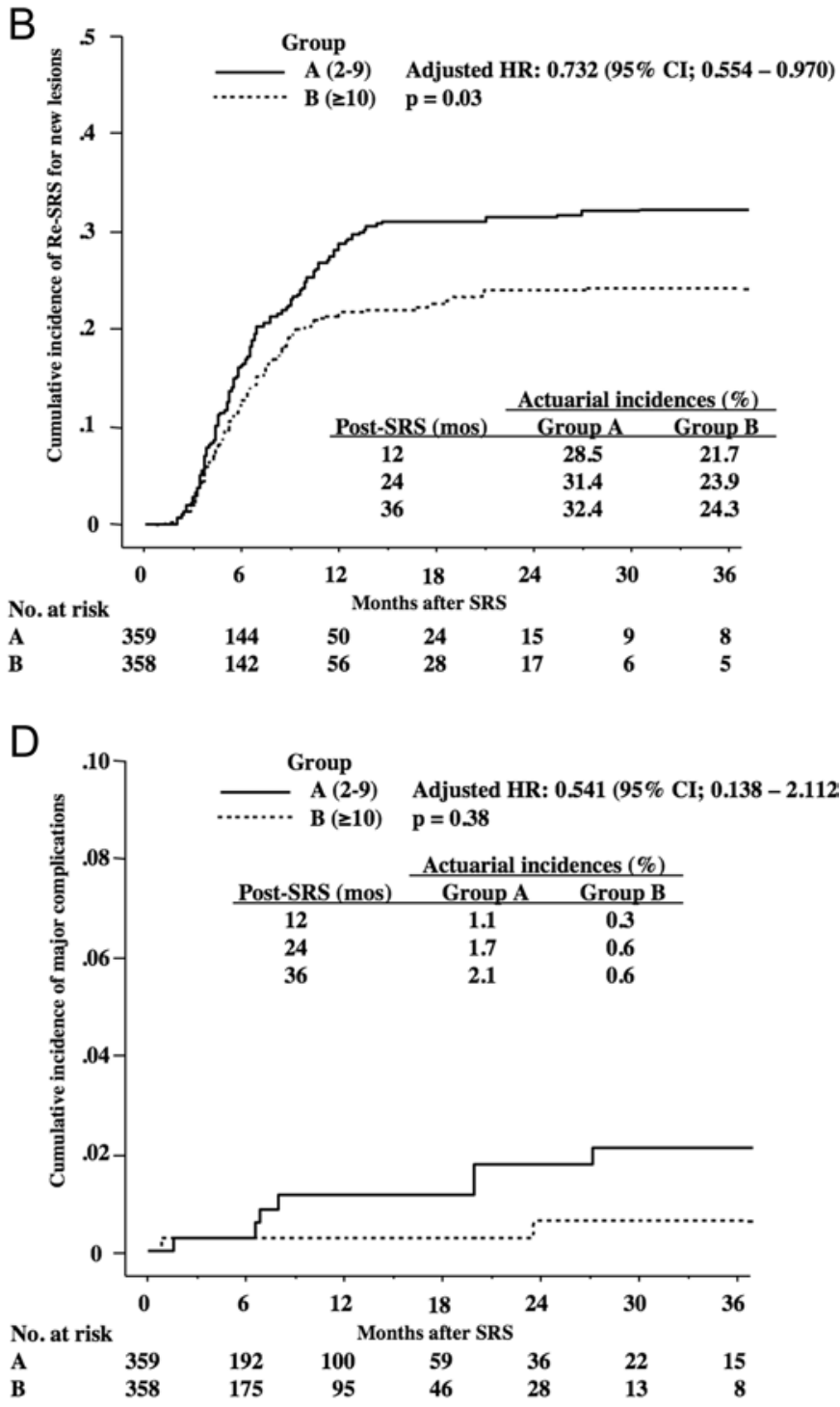

FIG. 2. Cumulative incidence of local recurrence (A), repeat SRS (Re-SRS) for new lesions (B), neurological deterioration (C), and major complications (D) according to number of tumors (2-9 [Group A] and 10 or more [Group B ]), estimated by using competing risk analysis. Three cases lost to follow-up were excluded. The estimated cumulative incidence of local recurrence was based on 485 (249 in Group A and 236 in Group B) patients (235 patients were excluded because neuroimaging results were not available).

al tumor adds approximately 10 minutes to the treatment time if the tumors are relatively small and a newly loaded unit is used. Therefore, for the past 1.5 decades, challenges have been encountered when using SRS for patients with 5 or more, or even 10 or more, metastatic brain tumors. $3,8,13,15,20,23,24,26,28-30,32$ For our cohort of 560 patients with 10 or more tumors, the post-SRS median survival time of 5.6 (95\% CI 4.9-6.4) months was slightly longer than the 4 months reported by Rava et al. ${ }^{20}$ but similar to the 6 months reported by Grandhi et al. ${ }^{8}$

The North American Gamma Knife Consortium is currently conducting a prospective randomized study entitled "A Randomized Controlled Study Of Neurocognitive Outcomes In Patients With Five Or More Brain Metastases Treated With Radiosurgery Or Whole-Brain Radiotherapy" (http://www.clinicaltrials.gov/, identifier
NCT01731704). The primary aim of that study is to compare the changes in neurocognitive function outcomes between baseline and 6 months among patients who undergo WBRT versus SRS. Patients with more than 4 metastatic brain tumors are eligible for this study. The results are expected to clarify the role of SRS alone versus WBRT.

\section{Is SRS Alone for Multiple Brain Metastases Safe?}

In 2002, the first author (M.Y.) and colleagues reported that in a series of 80 patients with 10 or more metastatic brain tumors (median 17, maximum 43) undergoing SRS, the estimated absorbed doses to the whole brain ranged from 2.16 to 8.51 (median 4.71) Gy. ${ }^{29}$ It was thus assumed that these doses had not exceeded the threshold level of radiation-induced injury to the whole brain. Kawabe et al. presented evidence, based on 1246 SRS procedures for 
TABLE 4: Univariable analysis of survival after SRS among 720 matched patients with brain metastases

\begin{tabular}{|c|c|c|c|c|}
\hline \multirow[b]{3}{*}{ Factor } & \multicolumn{4}{|c|}{ No. of Tumors } \\
\hline & \multicolumn{2}{|c|}{ 2-9 (Group A) } & \multicolumn{2}{|c|}{$\geq 10$ (Group B) } \\
\hline & $\operatorname{HR}(95 \% \mathrm{Cl})$ & $p$ Value & $\operatorname{HR}(95 \% \mathrm{Cl})$ & $\mathrm{p}$ Value \\
\hline no. of patients & 360 & & 360 & \\
\hline sex: M vs F & $1.309(1.054-1.630)$ & 0.01 & $1.351(1.090-1.677)$ & 0.006 \\
\hline \multicolumn{5}{|l|}{ age } \\
\hline continuous & $1.007(0.996-1.017)$ & 0.20 & $1.015(1.005-1.025)$ & 0.002 \\
\hline$>65$ vs $\leq 65$ yrs & $1.213(0.976-1.504)$ & 0.08 & $1.278(1.032-1.584)$ & 0.02 \\
\hline \multicolumn{5}{|l|}{ no. of tumors } \\
\hline continuous & $1.069(1.018-1.121)$ & 0.007 & $1.009(0.995-1.022)$ & 0.22 \\
\hline$\leq 13$ vs $\geq 14$ & & & $1.141(0.920-1.417)$ & 0.23 \\
\hline $\begin{array}{l}\text { primary cancer: lung vs not lung } \\
\text { extracerebral METs }\end{array}$ & $1.024(0.808-1.289)$ & 0.84 & $1.125(0.890-1.413)$ & 0.32 \\
\hline controlled vs not controlled & $2.726(2.112-3.550)$ & $<0.001$ & $2.402(1.856-3.108)$ & $<0.001$ \\
\hline present vs absent & $1.443(1.163-1.791)$ & $<0.001$ & $1.347(1.088-1.670)$ & 0.006 \\
\hline KPS score: $\geq 80 \%$ vs $\leq 70 \%$ & $2.140(1.666-1.725)$ & $<0.001$ & $3.206(2.467-4.168)$ & $<0.001$ \\
\hline \multicolumn{5}{|l|}{ modified-RPA class } \\
\hline I+Ila vs Ilb & $1.894(1.298-2.826)$ & $<0.001$ & $1.447(0.975-2.211)$ & 0.07 \\
\hline$\| b$ vs $\|c+\mid\|$ & 2.211 (1.728-2.849) & $<0.001$ & $2.451(1.926-3.136)$ & $<0.001$ \\
\hline neurological Sx: yes vs no & $1.412(1.139-1.750)$ & 0.002 & $1.201(0.970-1.487)$ & 0.09 \\
\hline prior surgery: yes vs no & $0.921(0.671-1.237)$ & 0.59 & 0.795 (0.579-1.092) & 0.15 \\
\hline prior WBRT: yes vs no & $0.888(0.540-1.371)$ & 0.61 & $1.061(0.631-1.783)$ & 0.82 \\
\hline \multicolumn{5}{|l|}{ tumor volume } \\
\hline cumulative & $1.016(1.008-1.023)$ & $<0.001$ & 1.023 (1.014-1.032) & $<0.001$ \\
\hline largest tumor & $1.028(1.014-1.041)$ & $<0.001$ & $1.032(1.018-1.045)$ & $<0.001$ \\
\hline peripheral dose & $0.954(0.921-0.987)$ & 0.007 & $0.938(0.901-0.978)$ & 0.003 \\
\hline
\end{tabular}

900 patients with 5 or more metastatic brain tumors, that total absorbed energy for the whole skull of 15 Joules is clearly safe but that total absorbed energy exceeding 20 Joules carries an unacceptably high risk of causing radiation-induced brain injury; the zone between lower and higher risk may thus exist somewhere between 15 and 20 Joules (Kawabe T, Yamamoto M, Barfod BE, Urakawa Y: Gamma knife radiosurgery for multiple brain metastases: what is a safe integral dose for the whole skull? Paper presented at the 10th Congress of International Stereotactic Radiosurgery Society; Paris, France; May 9, 2011). Furthermore, we also recently reported that among 167 brain metastasis patients who survived more than 3 years after SRS, the number of tumors did not affect the incidence of SRS-induced complications (HR 1.066, 95\% CI $0.968-1.131, \mathrm{p}=0.1567) .{ }^{31}$ Our herein-reported results show no apparent increased risk for complications with SRS for patients with 5 or more metastatic brain tumors compared with 4 or more metastatic brain tumors. PostSRS MR imaging confirmed the absence of leukoencephalopathy in patients who had undergone SRS alone. Also, in the study reported here, the cumulative incidence of SRS-induced complications among Group B patients was very similar to that among Group A patients. However, only patients with Radiation Therapy Oncology Group neurotoxicity Grade II or worse were counted in this study. ${ }^{19}$ Usually, physicians who manage individual cases do not report minor problems like neurotoxicity Grade 0 or 1 to us. Therefore, the weakness of this study is that all patients who experienced minor complications were not surveyed.

How Should Good Candidates for SRS Alone Be Selected From Among Patients With 10 or More Brain Metastases?

Grandhi et al. ${ }^{8}$ very recently reported that both univariable and multivariable analyses demonstrated that significant predictors of longer survival were tumor numbers of 13 or fewer versus 14 or more, melanoma versus other primary tumor types, better systemic disease status, and higher RPA class. Our database included only 2 melanoma patients. Thus, we could not test validity for melanoma versus other primary tumor types. In our study, we did not find the number of tumors, either as continuous or categorical variables (13 or fewer vs 14 or more), to be a significant predictor of survival duration (Table 4). Rather, our data indicate the following to favor a longer survival period: controlled primary cancer, no extracerebral metastases, better KPS scores, and higher RPA class. These factors were regarded as the 4 major prognostic factors for selecting good candidates.

\section{Study Weaknesses}

The major weakness of this study might be that be- 
cause our cohort included all treated patients, clinical factors are obviously heterogeneous. Greater patient group homogeneity makes a study more scientific. However, heterogeneity actually reflects clinical settings rather closely as we physicians often deal with clinical factors that are not homogeneous. Particularly, our database included some patients whose brain metastases were not newly diagnosed. However, proportions of such patients in the 2 groups were very small and did not differ significantly (Table 2). Thus, this heterogeneity had only a minimal effect on our results, as we have reported elsewhere. ${ }^{33}$

Treatment selection is considered to be largely influenced by the characteristics of patients receiving a particular therapeutic regimen. This issue is important when estimating the effect of treatments or exposures on outcomes by using observational data. One approach for reducing or eliminating the effect of treatment selection bias and confounding effects is to use propensity score matching, which enables one to design and analyze an observational (nonrandomized) study that mimics some of the characteristics of a randomized controlled trial. ${ }^{4}$ Original tumor phenotypes are now known to affect patient survival. Because these data were lacking for most patients who underwent SRS in the earlier years of our study period, we could not include them as a clinical factor for case matching.

\section{Conclusions}

Post-SRS treatment results (i.e., median survival time; neurological death-free survival time; and cumulative incidence of local recurrence, repeat SRS for new lesions, neurological deterioration, and SRS-related complications were not inferior among Group B patients when compared with those among Group A patients. We conclude that carefully selected patients with 10 or more metastatic brain tumors are not unfavorable candidates for SRS alone. However, a randomized controlled trial is necessary, in the near future, to clarify the most appropriate role for SRS alone in patients with 10 or more metastatic brain tumors.

\section{Acknowledgment}

We are very grateful to Bierta E. Barfod, Katsuta Hospital Mito GammaHouse, for her help in preparing this English manuscript.

\section{Disclosure}

The authors report no conflict of interest concerning the materials or methods used in this study or the findings specified in this paper.

Author contributions to the study and manuscript preparation include the following. Conception and design: Yamamoto. Acquisition of data: Yamamoto, Kawabe, Watanabe. Analysis and interpretation of data: Yamamoto, Kawabe, Nariai. Critically revising the article: Yamamoto. Reviewed submitted version of manuscript: Yamamoto, Kawabe, Sato, Higuchi, Nariai, Kasuya. Approved the final version of the manuscript on behalf of all authors: Yamamoto. Statistical analysis: Sato, Higuchi. Study supervision: Yamamoto, Kasuya.

\section{References}

1. Aoyama H, Shirato H, Tago M, Nakagawa K, Toyoda T, Hata- no K, et al: Stereotactic radiosurgery plus whole-brain radiation therapy vs stereotactic radiosurgery alone for treatment of brain metastases: a randomized controlled trial. JAMA 295:2483-2491, 2006

2. Bakoyannis G, Touloumi G: Practical methods for competing risks data: a review. Stat Methods Med Res 21:257-272, 2012

3. Chang WS, Kim HY, Chang JW, Park YG, Chang JH: Analysis of radiosurgical results in patients with brain metastases according to the number of brain lesions: is stereotactic radiosurgery effective for multiple brain metastases? Clinical article. J Neurosurg 113 Suppl:73-78, 2010

4. D'Agostino RB Jr: Propensity score methods for bias reduction in the comparison of a treatment to a non-randomized control group. Stat Med 17:2265-2281, 1998

5. Fine JP, Gray RJ: A proportional hazards model for the subdistribution of a competing risk. J Am Stat Assoc 94:496509, 1999

6. Gaspar L, Scott C, Rotman M, Asbell S, Phillips T, Wasserman $\mathrm{T}$, et al: Recursive partitioning analysis (RPA) of prognostic factors in three Radiation Therapy Oncology Group (RTOG) brain metastases trials. Int J Radiat Oncol Biol Phys 37:745751,1997

7. Gooley TA, Leisenring W, Crowley J, Storer BE: Estimation of failure probabilities in the presence of competing risks: new representations of old estimators. Stat Med 18:695-706, 1999

8. Grandhi R, Kondziolka D, Panczykowski D, Monaco EA III, Kano H, Niranjan A, et al: Stereotactic radiosurgery using the Leksell Gamma Knife Perfexion unit in the management of patients with 10 or more brain metastases. Clinical article. J Neurosurg 117:237-245, 2012

9. Gray RJ: A class of K-sample tests for comparing the cumulative incidence of a competing risk. Ann Stat 16:1141-1154, 1988

10. Hanssens P, Karlsson B, Yeo TT, Chou N, Beute G: Detection of brain micrometastases by high-resolution stereotactic magnetic resonance imaging and its impact on the timing of and risk for distant recurrences. Clinical article. J Neurosurg 115:499-504, 2011

11. Hayashi M, Yamamoto M, Nishimura C, Satoh H: Do recent advances in MR technologies contribute to better gamma knife radiosurgery treatment results for brain metastases? Neuroradiol J 20:481-490, 2007

12. Kaplan EL, Meier P: Nonparametric estimation from incomplete observations. J Am Stat Assoc 53:457-481, 1958

13. Karlsson B, Hanssens P, Wolff R, Söderman M, Lindquist C, Beute G: Thirty years' experience with Gamma Knife surgery for metastases to the brain. Clinical article. J Neurosurg 111:449-457, 2009

14. Karnofsky DA, Buechenal JH: The clinical evaluation of chemotherapeutic agents in cancer, in MacLeod CM (ed): Evaluation of Chemotherapeutic Agent. New York: Columbia University Press, 1949, pp 191-205

15. Kim CH, Im YS, Nam DH, Park K, Kim JH, Lee JI: Gamma knife radiosurgery for ten or more brain metastases. J Korean Neurosurg Soc 44:358-363, 2008

16. Knisely JPS, Yamamoto M, Gross CP, Castrucci WA, Jokura $\mathrm{H}$, Chiang VLS: Radiosurgery alone for 5 or more brain metastases: expert opinion survey. Clinical article. J Neurosurg 113 Suppl:84-89, 2010

17. Linskey ME, Andrews DW, Asher AL, Burri SH, Kondziolka $\mathrm{D}$, Robinson PD, et al: The role of stereotactic radiosurgery in the management of patients with newly diagnosed brain metastases: a systematic review and evidence-based clinical practice guideline. J Neurooncol 96:45-68, 2010

18. Parsons LS: Reducing bias in a propensity score matchedpair sample using greedy matching techniques. SAS. (http:// www2.sas.com/proceedings/sugi26/p214-26.pdf) [Accessed August 11, 2014] 


\section{Radiosurgery for $2-9$ versus 10 or more metastatic brain tumors}

19. Radiation Therapy Oncology Group: Cooperative Group Common Toxicity Criteria. (http://www.rtog.org/Research Associates/AdverseEventReporting/CooperativeGroup CommonToxicityCriteria.aspx) [Accessed August 13, 2014]

20. Rava P, Leonard K, Sioshansi S, Curran B, Wazer DE, Cosgrove GR, et al: Survival among patients with 10 or more brain metastases treated with stereotactic radiosurgery. Clinical article. J Neurosurg 119:457-462, 2013

21. Rosenbaum PR, Rubin DB: The central role of the propensity score in observational studies for causal effects. Biometrika 70:41-55, 1983

22. Satagopan JM, Ben-Porat L, Berwick M, Robson M, Kutler $\mathrm{D}$, Auerbach AD: A note on competing risks in survival data analysis. Br J Cancer 91:1229-1235, 2004

23. Serizawa T, Hirai T, Nagano O, Higuchi Y, Matsuda S, Ono J, et al: Gamma knife surgery for 1-10 brain metastases without prophylactic whole-brain radiation therapy: analysis of cases meeting the Japanese prospective multi-institute study (JLGK0901) inclusion criteria. J Neurooncol 98:163-167, 2010

24. Serizawa T, Yamamoto M, Sato Y, Higuchi Y, Nagano O, Kawabe T, et al: Gamma Knife surgery as sole treatment for multiple brain metastases: 2-center retrospective review of 1508 cases meeting the inclusion criteria of the JLGK0901 multi-institutional prospective study. Clinical article. J Neurosurg 113 Suppl:48-52, 2010

25. Sturm V, Kober B, Höver KH, Schlegel W, Boesecke R, Pastyr $\mathrm{O}$, et al: Stereotactic percutaneous single dose irradiation of brain metastases with a linear accelerator. Int J Radiat Oncol Biol Phys 13:279-282, 1987

26. Suzuki S, Omagari J, Nishio S, Nishiye E, Fukui M: Gamma knife radiosurgery for simultaneous multiple metastatic brain tumors. J Neurosurg 93 Suppl 3:30-31, 2000

27. Tsao MN, Rades D, Wirth A, Lo SS, Danielson BL, Gaspar LE, et al: Radiotherapeutic and surgical management for newly diagnosed brain metastasis(es): an American Society for Radiation Oncology evidence-based guideline. Pract Radiat Oncol 2:210-225, 2012

28. Yamamoto M, Ide M, Jimbo M, Aiba M, Ito M, Hirai H, et al: Gamma knife radiosurgery with numerous target points for intracranially disseminated metastases: early experience in three patients and experimental analysis of whole brain irradiation doses, in Kondziolka D (ed): Radiosurgery 1997. Basel: Karger, 1998, Vol 2, pp 94-109

29. Yamamoto M, Ide M, Nishio Si, Urakawa Y: Gamma Knife radiosurgery for numerous brain metastases: is this a safe treatment? Int J Radiat Oncol Biol Phys 53:1279-1283, 2002

30. Yamamoto M, Kawabe T, Barfod BE: How many metastases can be treated with radiosurgery? Prog Neurol Surg 25:261272, 2012

31. Yamamoto M, Kawabe T, Higuchi Y, Sato Y, Nariai T, Barfod $\mathrm{BE}$, et al: Delayed complications in patients surviving at least 3 years after stereotactic radiosurgery for brain metastases. Int J Radiat Oncol Biol Phys 85:53-60, 2013

32. Yamamoto M, Kawabe T, Sato Y, Higuchi Y, Nariai T, Barfod $\mathrm{BE}$, et al: A case-matched study of stereotactic radiosurgery for patients with multiple brain metastases: comparing treatment results for $1-4$ vs $\geq 5$ tumors. Clinical article. J Neurosurg 118: 1258-1268, 2013

33. Yamamoto M, Sato Y, Serizawa T, Kawabe T, Higuchi Y, Nagano $\mathrm{O}$, et al: Subclassification of recursive partitioning analysis Class II patients with brain metastases treated radiosurgically. Int J Radiat Oncol Biol Phys 83:1399-1405, 2012

34. Yamamoto M, Serizawa T, Sato Y, Kawabe T, Higuchi Y, Nagano $\mathrm{O}$, et al: Validity of two recently-proposed prognostic grading indices for lung, gastro-intestinal, breast and renal cell cancer patients with radiosurgically-treated brain metastases. J Neurooncol 111:327-335, 2013

35. Yamamoto M, Serizawa T, Shuto T, Akabane A, Higuchi Y, Kawagishi J, et al: Stereotactic radiosurgery for patients with multiple brain metastases (JLGK0901): a multi-institutional prospective study. Lancet Oncol 15:387-395, 2014

Manuscript submitted June 24, 2014.

Accepted August 4, 2014.

Please include this information when citing this paper: DOI: 10.3171/2014.8.GKS141421.

Address correspondence to: Masaaki Yamamoto, M.D., Katsuta Hospital Mito GammaHouse, 5125-2 Nakane, Hitachi-naka, Ibaraki 312-0011, Japan. email: bcd06275@nifty.com. 